\title{
Droplet motion with phase change in a temperature gradient
}

\section{$\operatorname{AUTHOR}(\mathrm{S}):$}

Onuki, A; Kanatani, K

\section{CITATION:}

Onuki, A ... [et al]. Droplet motion with phase change in a temperature gradient. Physical Review E 2005, 72(6): 066304.

\section{ISSUE DATE:}

2005-12

URL:

http://hdl.handle.net/2433/49913

RIGHT:

Copyright 2005 American Physical Society 
PHYSICAL REVIEW E 72, 066304 (2005)

\title{
Droplet motion with phase change in a temperature gradient
}

\author{
Akira Onuki and Kentaro Kanatani \\ Department of Physics, Kyoto University, Kyoto 606-8502, Japan
}

(Received 20 June 2005; published 9 December 2005)

\begin{abstract}
We examine the droplet motion in one-component fluids in a small temperature gradient by solving linearized hydrodynamic equations supplemented with appropriate surface boundary conditions. We show that the velocity field and the temperature around the droplet are strongly influenced by first-order phase transition taking place at the interface. Latent heat released or absorbed at the interface drastically changes the hydrodynamic flow around the droplet. As a result, the temperature becomes almost homogeneous inside the droplet and the Marangoni effect arising from the surface tension gradient is much suppressed. The droplet velocity is also much decelerated.
\end{abstract}

DOI: 10.1103/PhysRevE.72.066304

PACS number(s): 47.55.Dz, 68.03.Fg, 64.70.Fx, 44.30.+v

\section{INTRODUCTION}

Bubble motion in liquid is observed in everyday life, but its mathematical description is not simple [1]. As a classic example, the flow field around a spherical droplet moving at a constant velocity $v_{g}$ in gravity $g$ was calculated by Hadamard [2] and by Rybczynski [3] many years ago. To linear order in $g$ they found

$$
v_{g}=\frac{\left(\eta+\eta^{\prime}\right)\left(\rho-\rho^{\prime}\right)}{3 \eta\left(\eta+3 \eta^{\prime} / 2\right)} R^{2} g,
$$

where $\rho$ and $\eta\left(\rho^{\prime}\right.$ and $\left.\eta^{\prime}\right)$ are the mass density and the shear viscosity outside (inside) the droplet. Hereafter the quantities with (without) a prime are those inside (outside) the droplet. In their calculation no mass flux through the interface was assumed and no temperature deviation was involved. Under these assumptions the droplet shape remains nearly spherical when the Reynolds number $\operatorname{Re}=v_{g} R / \nu \sim g R^{3}\left(1-\rho^{\prime} / \rho\right) / \nu^{2}$ is small. Here $\nu=\eta / \rho$ is the exterior kinematic viscosity.

As another classic example, Young et al. [4] calculated the steady velocity of a spherical droplet in a small temperature gradient,

$$
\mathcal{T}=\left(\frac{d T}{d z}\right)_{\infty} .
$$

Hereafter $\mathcal{T}$ is the temperature gradient far from the droplet, where $\nabla T$ is along the $z$ axis. Still assuming no phase change at the interface, they took into account the dependence of the surface tension $\gamma$ on the deviation $\delta T=T-T_{0}$,

$$
\gamma=\gamma_{0}-\gamma_{1} \delta T
$$

where $T_{0}$ is a reference temperature and $\gamma_{1}=-d \gamma / d T$ is usually a positive constant. Then a droplet with radius $R$ moves with

$$
v_{\mathrm{Y}}=\frac{\lambda \gamma_{1}}{\left(\eta+3 \eta^{\prime} / 2\right)\left(2 \lambda+\lambda^{\prime}\right)} R \mathcal{T}
$$

to linear order in $\mathcal{T}$. The $\lambda\left(\lambda^{\prime}\right)$ is the thermal conductivity outside (inside) the droplet. This is an example of the Marangoni effect [1], where the motion occurs to lower the surface free energy. At zero gravity, the droplet migration is toward the warmer region both for gas and liquid droplets under $d \gamma / d T<0$. In gravity and in temperature gradient the migration velocity is $v_{\mathrm{Y}}+v_{g}$, where $v_{g}$ remains the same as that in Eq. (1.1) (even if heat conduction is included in the calculation). Note that Eq. (1.4) is justified for small Reynolds number $\mathrm{Re}$ and small Marangoni number $\mathrm{Ma}=R^{2} \gamma_{1} \mathcal{T} / \eta D_{T}$ [5], where $D_{T}=\lambda / \rho C_{p}$ is the (exterior) thermal diffusion constant with $C_{p}$ being the isobaric specific heat per unit mass. Young et al. also reported an experiment of applying large temperature gradients of order $10-10^{2} \mathrm{~K} / \mathrm{cm}$, which balanced with the buoyancy and held bubbles stationary [4].

Equation (1.4) can be used only when the gas and the liquid consist of different fluids without phase change, for example, in the case of air bubbles in silicone oil $[4,6,7]$. However, in some recent experiments, droplets were those produced in phase separation in a binary mixture [8] and in $\mathrm{CO}_{2}$ [9]. In such fluids first-order phase transition between the two phases (evaporation and condensation) can occur at the interface, but this effect has not yet been examined in the literature. This paper aims to construct a first theory of this effect in one-component fluids.

If phase change is allowed, the fluid regions near the interfaces should be close to the coexistence curve $T=T_{\mathrm{cx}}(p)$ in the $p-T$ phase diagram. In zero gravity, the temperature gradient should then nearly vanish inside each droplet, since the pressure is homogeneous outside the droplets (and is slightly higher inside the droplets by $2 \gamma / R$ ) in slowly evolving states with $\operatorname{Re} \ll 1$. Recently such temperature profiles have been obtained numerically in a dynamic van der Waals theory [10]. Although it is a natural consequence in one-component fluids without contamination $[11,12]$, the mechanism is dynamical and is hence highly nontrivial. We point out that a convective velocity of order

$$
v_{c}=\frac{q}{\rho^{\prime} T \Delta s}=-\frac{\lambda \mathcal{T}}{\rho^{\prime} T \Delta s}
$$

carrying latent heat $T \Delta s$ is sufficient to suppress the temperature gradient inside a droplet. Here $q=-\lambda \mathcal{T}$ is the applied heat flux, and $\Delta s=s^{\prime}-s$ is the entropy difference per unit mass. At zero gravity, we shall see that the droplet migration 
velocity $v_{\mathrm{D}}$ is of order $v_{c}$. For a gas droplet in $\mathrm{CO}_{2}$ at $T=298 \mathrm{~K}$, for example, we have $v_{c} \sim 0.74 \times 10^{-2} \mathcal{T} / T \mathrm{~cm} / \mathrm{s}$ with $\mathcal{T} / T$ in $\mathrm{cm}^{-1}$ [13]. It is of interest to compare the two characteristic velocities, $v_{\mathrm{Y}}$ in Eq. (1.4) and $v_{c}$ in Eq. (1.5). To this end we introduce the dimensionless number,

$$
M_{1}=R \gamma_{1} \rho^{\prime} T \Delta s / \eta \lambda,
$$

in terms of which $v_{c} / v_{\mathrm{Y}} \sim M_{1}^{-1}$ for a gas droplet. As very rough estimates for simple fluids far from the critical point, we set $[14,15]$

$$
D_{T}=\frac{\lambda}{\rho C_{p}} \sim \frac{k_{\mathrm{B}} T}{6 \pi \eta a}, \quad \Delta s \sim C_{p}, \quad \gamma_{1} \sim \frac{k_{\mathrm{B}}}{a^{2}},
$$

where $a$ is a microscopic length of the order of the atomic radius. This leads to $M_{1} \sim \rho^{\prime} R / \rho a$. Thus $M_{1} \gg 1$ and $\left|v_{c} / v_{\mathrm{Y}}\right| \ll 1$ for large $R$. See Sec. IV D for the behavior of $M_{1}$ near the critical point. For a liquid droplet in gas, on the other hand, we have $v_{c} / v_{\mathrm{Y}} \sim \eta^{\prime} \lambda^{\prime} / \eta \lambda M_{1}$. With increasing $R$ the highly efficient latent heat transport should readily dominate over the usual heat conduction within a droplet. This indicates vanishing of the surface tension variation along the interface and suppression of the Marangoni effect, against heat flow, in one component fluids.

This paper will present linear analysis of the simplest case of a spherical droplet in weak gravity and temperature gradient. In Sec. II we will give linear hydrodynamic equations and surface boundary conditions. In Sec. III we will examine the axisymmetric situations. In Sec. IV we will give some new predictions, which could be verified particularly in the weightless condition. Estimations near the critical point will be presented.

\section{BASIC EQUATIONS}

\section{A. Spherical droplet}

We consider a gas or liquid droplet in a one-component fluid. In Appendix A we will examine how such a droplet can be stable theoretically and be prepared experimentally. In the unperturbed equilibrium state described in Appendix A, the pressure $p$ is a constant $p_{0}$ for $r>R$ and is $p_{0}+2 \gamma_{0} / R$ for $r<R$, the temperature is homogeneous $\left(T=T_{0}\right)$, and there is no fluid motion $(\boldsymbol{v}=\mathbf{0})$. The pressure inside the droplet is higher than that outside it by $2 \gamma_{0} / R$ (Laplace's law). We then apply a temperature gradient $\mathcal{T}=(d T / d z)_{\infty}$ and a gravity field $g$ and assume that the droplet is moving at a constant velocity $v_{\infty}$ in the vertical $z$ axis. We may take the origin of the reference frame at the droplet center and seek a steady axisymmetric solution of the hydrodynamic equations with appropriate boundary conditions. For small $\mathcal{T}=(d T / d z)_{\infty}$ and $g$ and/or for small $R$, the droplet shape may be assumed to be spherical, as in the previous theories [2-4]. Deviation from sphericity occurs from second orders in $\mathcal{T}$ or $g$ [16].

In this paper the time derivatives and contributions of order $\mathcal{T}^{2}, \mathcal{T} g$, or $g^{2}$ in the hydrodynamic equations will be neglected. We employ these assumptions for the mathematical simplicity, though they are very restrictive. To apply our theory, we suppose space experiments for a macroscopically large gas droplet, while a very tiny droplet needs to be used under the earth gravity. For a liquid droplet in gas far from the critical point, the viscosity of the surrounding gas is very low [17] and gravity-induced acceleration is unavoidable and, even in space, it should be difficult to remove nonsteady fluid motions in heat flow. See the item (iii) in the last section for more comments.

In the following calculation it is convenient to use the spherical coordinates $(r, \theta, \varphi)$. The distance from the droplet center is written as $r$. Using the solid angles $\theta$ and $\varphi$ we define the three orthogonal unit vectors,

$$
\begin{gathered}
\boldsymbol{e}_{1}=r^{-1} \boldsymbol{r}=(\sin \theta \cos \varphi, \sin \theta \sin \varphi, \cos \theta), \\
\boldsymbol{e}_{2}=\frac{\partial}{\partial \theta} \boldsymbol{e}_{1}=(\cos \theta \cos \varphi, \cos \theta \sin \varphi,-\sin \theta), \\
\boldsymbol{e}_{3}=\boldsymbol{e}_{1} \times \boldsymbol{e}_{2}=(-\sin \varphi, \cos \varphi, 0) .
\end{gathered}
$$

\section{B. Linear hydrodynamic equations}

First we present the linearized hydrodynamic equations for $\boldsymbol{v}, \delta p$, and $\delta T[18]$ in steady states in the bulk region, which are valid both within and outside the droplet $(r<R$ and $r>R$ ). The continuity equation for the mass density $\rho$ yields

$$
\nabla \cdot(\rho \boldsymbol{v})=\rho \nabla \cdot \boldsymbol{v}+\boldsymbol{v} \cdot \nabla \rho=0 .
$$

Here $\partial \rho / \partial t=0$ is assumed and $\boldsymbol{v} \cdot \nabla \rho$ is of second order (of order $\mathcal{T} g$ in the gravity-induced density stratification) for $r$ $\neq R$. It follows the incompressibility condition,

$$
\boldsymbol{\nabla} \cdot \boldsymbol{v}=0
$$

even in compressible fluids. The momentum equation becomes

$$
-\nabla \delta p+\eta \nabla^{2} \boldsymbol{v}-\rho g \boldsymbol{e}_{z}=\mathbf{0},
$$

where $\boldsymbol{e}_{z}$ is the unit vector along the $z$ axis. In this equation the density deviation $\delta \rho$ induced by gravity and/or heat flow will be neglected so that the buoyancy effect arises from the discontinuous change in the mass density $\rho$ across the interface [see Eq. (3.4)]. The pressure deviation is defined by

$$
\begin{aligned}
\delta p & =p-p_{0} \quad(r>R) \\
& =p-p_{0}-2 \gamma_{0} / R \quad(r<R)
\end{aligned}
$$

Taking the divergence of Eq. (2.4) yields

$$
\nabla^{2} \delta p=0
$$

where we have neglected the term $-(\partial \rho / \partial z) g$ in the bulk region $(r \neq R)$. Also the temperature deviation $\delta T$ satisfies

$$
\lambda \nabla^{2} \delta T=0 .
$$

Supposing slow fluid motions we have neglected the nonlinear convective terms, $-\boldsymbol{\rho} \boldsymbol{v} \cdot \nabla \boldsymbol{v}$ in Eq. (2.4) and $-\rho \boldsymbol{v} \cdot \nabla s$ in Eq. (2.7) with $s$ being the entropy variable [18].

Notice that the coefficients $\eta, \rho$, and $\lambda$ in the above equations are different between the two phases. Therefore, they will be written as $\eta^{\prime}, \rho^{\prime}$, and $\lambda^{\prime}$ inside the droplet, while those outside it will remain unprimed. 


\section{Boundary conditions}

Next we consider the boundary conditions at the surface $r=R$. In the following equations Eqs. (2.8)-(2.17) the variables $\boldsymbol{v}, \delta T$, and $\delta p$ denote the values at $r=R+0$ (immediately outside the droplet), while $\boldsymbol{v}^{\prime}, \delta T^{\prime}$, and $\delta p^{\prime}$ denote those at $r=R-0$ (immediately inside the droplet).

(i) First we consider the boundary conditions imposed on the velocity field and those from the stress balance. The mass conservation at the surface yields

$$
J=\rho e_{1} \cdot v=\rho^{\prime} e_{1} \cdot v^{\prime},
$$

where $J$ is the mass flux through the interface representing conversion between gas and liquid. We assume the continuity of the tangential velocity,

$$
\boldsymbol{e}_{2} \cdot \boldsymbol{v}=\boldsymbol{e}_{2} \cdot \boldsymbol{v}^{\prime}
$$

The stress balance at the interface yields

$$
\begin{gathered}
\delta p-\eta e_{1} \cdot \overleftrightarrow{e} \cdot e_{1}-\frac{2}{R} \gamma_{1} \delta T=\delta p^{\prime}-\eta^{\prime} \boldsymbol{e}_{1} \cdot \overleftrightarrow{e^{\prime}} \cdot e_{1}, \\
-\eta e_{2} \cdot \overleftrightarrow{e} \cdot e_{1}+\gamma_{1} e_{2} \cdot \nabla \delta T=-\eta^{\prime} e_{2} \cdot \overleftrightarrow{e} \cdot e_{1},
\end{gathered}
$$

in the normal and tangential directions, respectively, where

$$
\overleftrightarrow{e}=\left\{\partial v_{i} / \partial x_{j}+\partial v_{j} / \partial x_{i}\right\}
$$

is the strain rate tensor. Equation (2.10) represents the boundary condition that the discontinuity of the tangential stress is equal to the tangential gradient of the surface tension, which arises from the momentum balance at the surface $[1,4,19]$.

(ii) Second, the energy conservation at the surface gives

$$
J_{e}=h J-e_{1} \cdot \lambda \nabla \delta T=h^{\prime} J-e_{1} \cdot \lambda^{\prime} \nabla \delta T^{\prime},
$$

where $J_{e}$ is the energy flux through the interface and

$$
h=(e+p) / \rho=T s+\mu
$$

is the enthalpy per unit mass. Here $e$ is the energy density, $s$ is the entropy per unit mass, and $\mu$ is the chemical potential per unit mass. Note that the energy current density is written as $\boldsymbol{J}_{e}=\rho h \boldsymbol{v}-\lambda \nabla T$ in the bulk region.

(iii) Third, we assume the continuity of the temperature and the chemical potential at the interface,

$$
\begin{gathered}
\delta T=\delta T^{\prime}, \\
\delta \mu=\delta \mu^{\prime}
\end{gathered}
$$

Using the Gibbs-Duhem relation $\delta \mu=-s \delta T+\rho^{-1} \delta p$ we rewrite Eq. (2.16) as

$$
-\Delta s \delta T=\frac{1}{\rho} \delta p-\frac{1}{\rho^{\prime}} \delta p^{\prime},
$$

where $\Delta s=s^{\prime}-s$. However, general statistical-mechanical theory shows that $T$ and $\mu$ can be discontinuous across an interface in the presence of $J$ and $J_{e}[19,20]$. Temperature discontinuity across an interface of evaporating liquid has been calculated by kinetic theory $[21,22]$ and has been observed in experiments [23]. In addition, in helium systems at low temperatures $[24,25]$, discontinuity of the chemical potential gives rise to a surface dissipation mechanism relevant to capillary-wave damping. In Appendix B, surface kinetic relations will be introduced, which are more general than Eqs. (2.15) and (2.16) and account for discontinuous changes of $T$ and $\mu$.

In the previous theories without first order phase transition [2-4], there was no mass conversion $(J=0)$ and the chemical equilibrium Eq. (2.16) was not assumed.

\section{AXISYMMETRIC SITUATIONS}

The gravity and the heat flux far from the droplet are assumed to be along the $z$ axis. Then the fluid flow is axisymmetric with respect to the $z$ axis.

\section{A. Expressions in the bulk region}

We first show the expressions for $\boldsymbol{v}, \delta p$, and $\delta T$ in the bulk region. As will be shown in Appendix C, the velocity field is expressed as [26]

$$
\begin{aligned}
\boldsymbol{v}(\boldsymbol{r}) & =\nabla[\hat{H}(r) \cos \theta]+[\hat{Q}(r) \cos \theta] \boldsymbol{r} \\
& =\left(\hat{H}^{\prime}-\frac{1}{r} \hat{H}+\hat{Q} r\right) \frac{z}{r^{2}} \boldsymbol{r}+\frac{1}{r} \hat{H} \boldsymbol{e}_{z},
\end{aligned}
$$

where $\cos \theta=z / r$ and $\boldsymbol{e}_{z}$ is the unit vector along the $z$ axis. Here $\hat{Q}$ and $\hat{H}$ depend only on $r$. Outside the droplet we have

$$
\hat{Q}=Q_{1} \frac{R}{r^{2}}, \quad \hat{H}=\frac{R}{2} Q_{1}+H_{1} \frac{R^{3}}{r^{2}}-v_{\mathrm{D}} r \quad(r>R) .
$$

Inside the droplet we have

$$
\hat{Q}=Q_{2}^{\prime} \frac{r}{R^{2}}, \quad \hat{H}=-\frac{2}{5 R^{2}} Q_{2}^{\prime} r^{3}+H_{2}^{\prime} r \quad(r<R) .
$$

Here $Q_{1}, H_{1}, v_{\mathrm{D}}, Q_{2}^{\prime}$, and $H_{2}^{\prime}$ are constants having the dimension of velocity. Far from the droplet $(r \gg R)$ we find $\boldsymbol{v} \rightarrow-v_{\mathrm{D}} \boldsymbol{e}_{z}$, so $v_{\mathrm{D}}$ is the droplet velocity in the original reference frame. A well-known example of the velocity field around a hard sphere can also be expressed in the form of Eq. (3.1) with Eq. (3.2) [27]. From Eq. (2.6) the pressure deviation is determined as

$$
\begin{aligned}
\delta p & =\eta Q_{1} \frac{R z}{r^{3}}-g \rho z \quad(r>R) \\
& =-2 \eta^{\prime} Q_{2}^{\prime} \frac{z}{R^{2}}-g \rho^{\prime} z \quad(r<R) .
\end{aligned}
$$

From Eq. (2.7) the temperature deviation is written as

$$
\begin{aligned}
\delta T & =\left(\mathcal{T}-\mathcal{T}_{1}\right) \frac{R^{3}}{2 r^{3}} z+\mathcal{T}_{z} \quad(r>R) \\
& =\mathcal{T}_{1}^{\prime} z \quad(r<R) .
\end{aligned}
$$

Here $\mathcal{T}_{1} \cos \theta=e_{1} \cdot \nabla T$ at $r=R+0, \mathcal{T}_{1} \cos \theta=e_{1} \cdot \nabla T$ at $r=R-0$, 
and $\mathcal{T}$ is the temperature gradient far from the droplet. The temperature gradient is homogeneous within the droplet:

$$
\left(\frac{d T}{d z}\right)_{\text {in }}=\mathcal{T}_{1}^{\prime}
$$

\section{B. Axisymmetric boundary conditions}

From the boundary conditions in Eqs. (2.9)-(2.17) we obtain linear relations among the coefficients $Q_{1}, H_{1}, v_{\mathrm{D}}, Q_{2}^{\prime}$, $H_{2}^{\prime}, \mathcal{T}_{1}$, and $\mathcal{T}_{1}$. The mass and energy fluxes through the interface are angle-dependent as

$$
J=J_{1} \cos \theta, \quad J_{e}=J_{1 e} \cos \theta,
$$

where $J_{1}$ and $J_{1 e}$ are constants.

(i) From Eqs. (2.8)-(2.11) we obtain

$$
\begin{gathered}
J_{1}=\rho\left[Q_{1}-2 H_{1}-v_{\mathrm{D}}\right]=\rho^{\prime}\left[H_{2}^{\prime}-\frac{1}{5} Q_{2}^{\prime}\right], \\
\frac{1}{2} Q_{1}+H_{1}-v_{\mathrm{D}}=-\frac{2}{5} Q_{2}^{\prime}+H_{2}^{\prime}, \\
\frac{3}{2} \eta\left(Q_{1}-4 H_{1}\right)+\frac{3}{5} \eta^{\prime} Q_{2}^{\prime}-\frac{g}{2} R^{2}\left(\rho-\rho^{\prime}\right)=R \gamma_{1} \mathcal{T}_{1}^{\prime}, \\
-6 \eta H_{1}+\frac{3}{5} \eta^{\prime} Q_{2}^{\prime}=R \gamma_{1} \mathcal{T}_{1}^{\prime} .
\end{gathered}
$$

As the temperature and the tangential temperature gradient at the interface in Eqs. (2.10) and (2.11) we have used the values at $r=R-0$. Equations (3.10) and (3.11) give

$$
Q_{1}=g R^{2}\left(\rho-\rho^{\prime}\right) / 3 \eta
$$

From these equations $H_{1}, v_{\mathrm{D}}$, and $H_{2}^{\prime}$ may be expressed in terms of $J_{1}, Q_{2}^{\prime}$, and $Q_{1}$ as

$$
\begin{gathered}
H_{1}=\frac{1}{3}\left(\frac{1}{\rho^{\prime}}-\frac{1}{\rho}\right) J_{1}-\frac{1}{15} Q_{2}^{\prime}+\frac{1}{6} Q_{1}, \\
v_{\mathrm{D}}=-\frac{1}{3}\left(\frac{2}{\rho^{\prime}}+\frac{1}{\rho}\right) J_{1}+\frac{2}{15} Q_{2}^{\prime}+\frac{2}{3} Q_{1}, \\
H_{2}^{\prime}=\frac{1}{\rho^{\prime}} J_{1}+\frac{1}{5} Q_{2}^{\prime},
\end{gathered}
$$

The interior temperature gradient $\mathcal{T}_{1}$ is expressed as

$$
R \gamma_{1} \mathcal{T}_{1}^{\prime}=\left(\frac{2}{5} \eta+\frac{3}{5} \eta^{\prime}\right) Q_{2}^{\prime}-2 \eta\left(\frac{1}{\rho^{\prime}}-\frac{1}{\rho}\right) J_{1}-\eta Q_{1}
$$

Now $J_{1}$ and $Q_{2}^{\prime}$ remain undetermined.

(ii) The energy conservation relation (2.13) leads to

$$
J_{e 1}=h J_{1}-\lambda \mathcal{T}_{1}=h^{\prime} J_{1}-\lambda^{\prime} \mathcal{T}_{1}^{\prime} .
$$

Next the temperature continuity Eq. (2.15) yields

$$
\mathcal{T}_{1}=3 \mathcal{T}-2 \mathcal{T}_{1}^{\prime},
$$

under which the tangential temperature gradient turns out to be commonly given by $\boldsymbol{e}_{2} \cdot \nabla T=-\mathcal{T}_{1} \sin \theta$ inside and outside the droplet $(r=R \pm 0)$. This means that there is no ambiguity in Eqs. (3.10) and (3.11) under the temperature continuity. From Eq. (3.17) we also obtain

$$
T \Delta s J_{1}=\left(2 \lambda+\lambda^{\prime}\right) \mathcal{T}_{1}^{\prime}-3 \lambda \mathcal{T},
$$

where $h^{\prime}-h=T\left(s^{\prime}-s\right)$ from the continuity of the chemical potential in Eq. (2.16). Equation (2.17) also gives

$$
\rho^{\prime} \Delta s \mathcal{T}_{1}^{\prime}=-\frac{\rho^{\prime} \eta}{\rho R^{2}} Q_{1}-\frac{2 \eta^{\prime}}{R^{2}} Q_{2}^{\prime} .
$$

Addition of Eqs. (3.19) and (3.20) to Eqs. (3.13)-(3.16) gives a complete solution.

\section{SOLUTIONS}

To simplify the mathematical expressions to follow, we use the following symbols:

$$
\hat{\rho}=\rho^{\prime} / \rho, \quad \hat{\eta}=\eta^{\prime} / \eta, \quad \hat{\lambda}=\lambda^{\prime} / \lambda .
$$

These numbers are smaller than 1 for a gas droplet in liquid and larger than 1 for a liquid droplet in gas [17].

\section{A. Case of $\mathcal{T} \neq 0$ and $g=0$ without phase change}

The results by Young et al. [4] can be obtained by setting $J_{1}=0$ in Eqs. (3.13)-(3.16) and (3.19), where the chemical equilibrium condition (3.20) is not used. They showed $v_{\mathrm{D}}=v_{\mathrm{Y}}$, where $v_{\mathrm{Y}}$ is given by Eq. (1.4). However, they did not show the explicit expression for the velocity field. It is simply given by

$$
\begin{aligned}
\boldsymbol{v}+v_{\mathrm{D}} \boldsymbol{e}_{z} & =-\frac{v_{\mathrm{D}}}{2} R^{3}\left[\frac{1}{r^{3}} \boldsymbol{e}_{z}-\frac{3 z}{r^{5}} \boldsymbol{r} \quad(r>R)\right. \\
& =v_{\mathrm{D}}\left[\frac{5}{2} \boldsymbol{e}_{z}+\frac{3 z}{2 R^{2}} \boldsymbol{r}-\frac{3 r^{2}}{R^{2}} \boldsymbol{e}_{z}\right] \quad(r<R) .
\end{aligned}
$$

For $r<R$ the velocity is a dipolar field. At $r=R$ it is continuous as $\boldsymbol{e}_{1} \cdot \boldsymbol{v}=0$ (or $\left.J=0\right)$ and $\boldsymbol{e}_{2} \cdot \boldsymbol{v}=-\left(3 H_{2} / 2\right) \sin \theta$. In Fig. 1 we display the above velocity field in the $x-z$ plane. The temperature deviation is of the form,

$$
\begin{aligned}
\delta T & =\left[\left(\frac{1-\hat{\lambda}}{2+\hat{\lambda}}\right) \frac{R^{3}}{r^{3}}+1\right] z \mathcal{T} \quad(r>R) \\
& =\frac{3}{2+\hat{\lambda}} z \mathcal{T} \quad(r<R) .
\end{aligned}
$$

Here the surface tension gradient in the tangential stress balance in Eq. (2.11) is crucial, resulting in the velocity of order $\gamma_{1}|\mathcal{T}| / \eta$. The temperature gradient within the droplet is along the $z$ axis and is equal to $3 \lambda \mathcal{T} /\left(2 \lambda+\lambda^{\prime}\right)$, which tends to $3 \mathcal{T} / 2$ for $\hat{\lambda} \rightarrow 0$. In Fig. 2 we show the normalized temperature deviation $[T(\boldsymbol{r})-T(\mathbf{0})] / R \mathcal{T}$ for $\hat{\lambda}=0.1$ in the $x-z$ plane.

\section{B. Case of $\mathcal{T} \neq 0$ and $g=0$ with phase change}

We proceed to the case with phase change. Here we assume $\mathcal{T} \neq 0$ and $g=0$. In addition to $M_{1}$ in Eq. (1.6) we introduce 


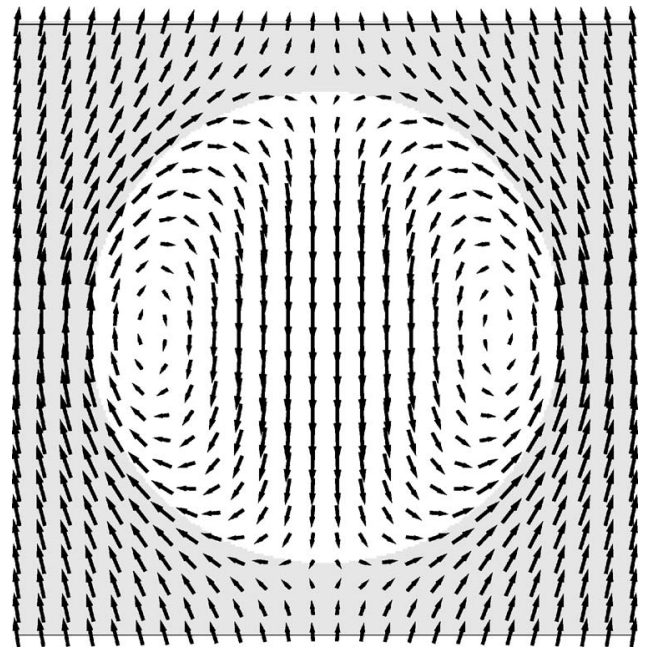

FIG. 1. Velocity field $\left(v_{x}, v_{z}\right)$ in a small temperature gradient without phase change in the reference frame moving with a droplet [4]. The velocity is tangential and continuous at the interface.

$$
M_{2}=R \rho^{\prime} \Delta s / \gamma_{1}
$$

From Eq. (1.7) it is also a large number of order $\rho^{\prime} R / \rho a$ with $a$ being a microscopic length far from the critical point.

(i) It is straightforward to solve Eqs. (3.13)-(3.20) with $Q_{1}=0$. Though complicated, $J_{1}, \mathcal{T}_{1}$, and $v_{\mathrm{D}}$ are expressed as

$$
\begin{gathered}
J_{1}=3 \rho^{\prime} v_{c} /\left[1+(2+\hat{\lambda}) \epsilon_{1}\right], \\
\mathcal{T}_{1}^{\prime}=3 \epsilon_{1} \mathcal{T} /\left[1+(2+\hat{\lambda}) \epsilon_{1}\right], \\
v_{\mathrm{D}}=-v_{c} \frac{\left(\hat{\rho}+M_{1} \epsilon_{1} / 3\right)+\hat{\eta}(1+\hat{\rho} / 2)}{\left[1+(2+\hat{\lambda}) \epsilon_{1}\right](1 / 3+\hat{\eta} / 2)},
\end{gathered}
$$

where $\epsilon_{1}$ is a small dimensionless number defined by

$$
\epsilon_{1}=\frac{10 \hat{\eta}(1-\hat{\rho})}{M_{1}\left[M_{2}(1+3 \hat{\eta} / 2)+5 \hat{\eta}\right]}
$$

and $v_{c}$ is the characteristic velocity in Eq. (1.5). Since $v_{\mathrm{D}} \sim-v_{c}$, a gas (liquid) droplet moves towards the warmer (cooler) region.

(ii) Simpler results can be obtained for large $M_{1}$ and $M_{2}$. From Eqs. (4.7) and (4.8) the temperature gradient $\mathcal{T}_{1}$ inside the droplet becomes very small compared to $\mathcal{T}$ as

$$
\left(\frac{d T}{d z}\right)_{\text {in }}=\mathcal{T}_{1} \cong \frac{10 \hat{\eta}(1-\hat{\rho})}{(1 / 3+\hat{\eta} / 2) M_{1} M_{2}} \mathcal{T}
$$

where $M_{1} M_{2}=R^{2}\left(\rho^{\prime} \Delta s\right)^{2} T / \eta \lambda$ and $(d T / d z)_{\text {in }} \propto \mathcal{T} / R^{2}$. In Appendix $\mathrm{B}$ another behavior $(d T / d z)_{\text {in }} \propto \mathcal{T} / R$ will emerge in the presence of the surface dissipation effect, which is also very small. The internal temperature gradient $(d T / d z)_{\text {in }}$ is virtually zero except for extremely tiny droplets.

In the following we give expressions in the limit of $M_{1} \rightarrow \infty$ and $M_{2} \rightarrow \infty$. From Eqs. (4.6) and (3.17) the fluxes through the interface are calculated as

$$
\begin{gathered}
J_{1} \cong 3 \rho^{\prime} v_{c}, \\
J_{e 1} \cong h^{\prime} J_{1} \cong\left(3 h^{\prime} / T \Delta s\right) q,
\end{gathered}
$$

where $v_{c}$ is defined by Eq. (1.5), $h^{\prime}$ is the enthalpy per unit mass within the droplet, and $q=-\lambda \mathcal{T}$ is the applied heat flux. The energy current is $h^{\prime} J_{1} \boldsymbol{e}_{z}$ within the droplet and the energy flux through the interface is $h^{\prime} J_{1} \cos \theta$. If we calculate the energy flux outside the droplet close to the interface, it consists of the convective term $h J_{1} \cos \theta$ and the heat conduction term $3 q \cos \theta$, with the sum being equal to the energy flux calculated within the droplet from $h^{\prime}-h=T \Delta s$. The droplet velocity is of the form,

$$
v_{\mathrm{D}} \cong \frac{\hat{\eta}+(1+\hat{\eta} / 2) \hat{\rho}}{(1 / 3+\hat{\eta} / 2) \rho^{\prime} T \Delta s} \lambda \mathcal{T} .
$$

The temperature is homogeneous inside the droplet and

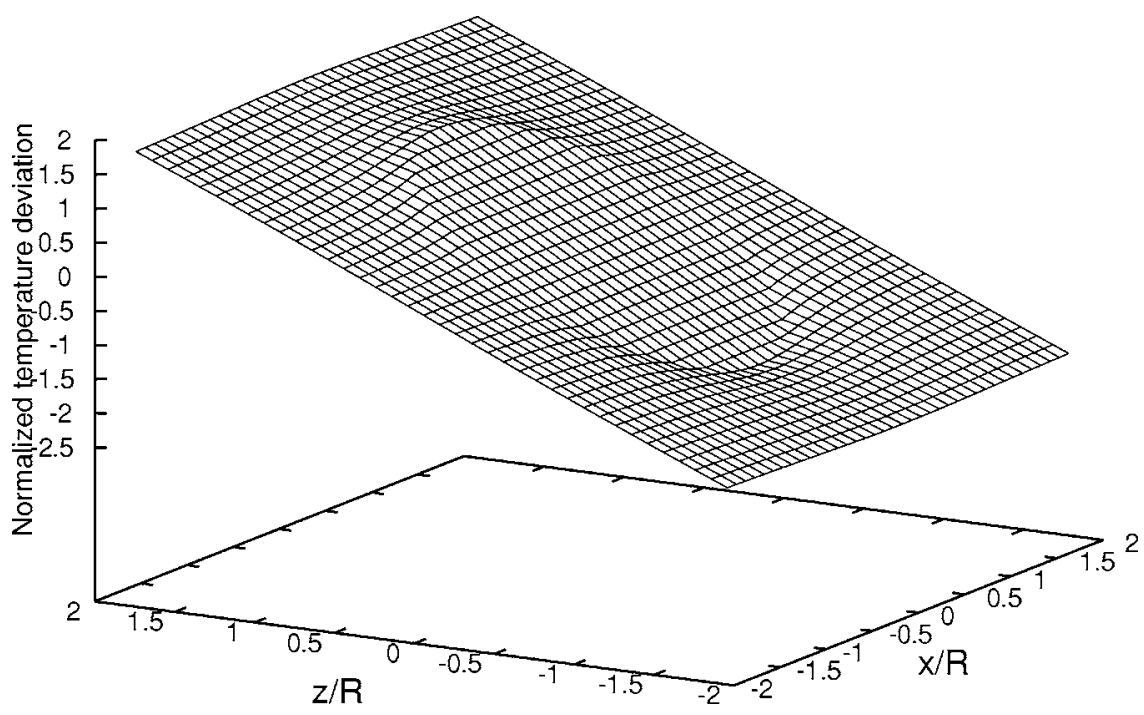

066304-5
FIG. 2. Normalized temperature deviation $[T(\boldsymbol{r})-T(\mathbf{0})] / R \mathcal{T}$ without phase change for the gas droplet case with $\hat{\lambda}=0.1$ in the $x-z$ plane. Its gradient within the droplet is larger than the applied gradient by $3 / 2.1$ from Eq. (4.3). 


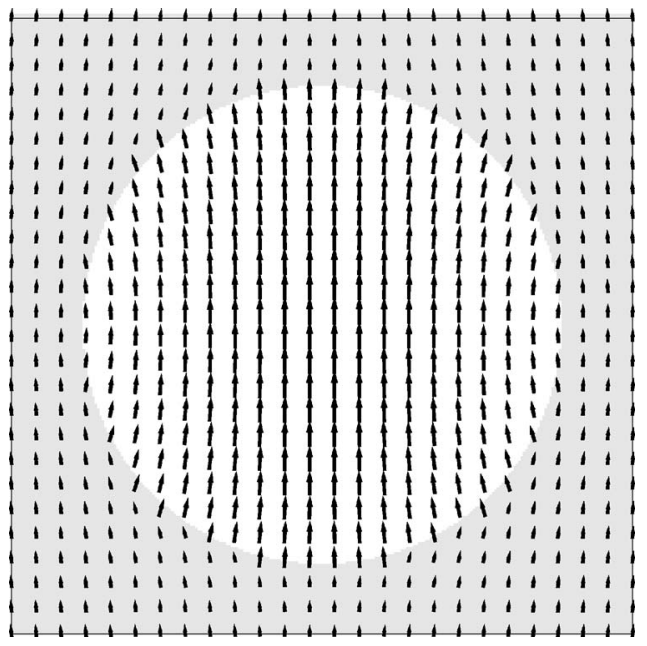

FIG. 3. Velocity field $\left(v_{x}, v_{z}\right)$ around a gas droplet in a small temperature gradient with phase change in the reference frame moving with the droplet. Here $\rho^{\prime} / \rho=\eta^{\prime} / \eta=0.3$ and use is made of Eq. (4.14).

$$
\delta T \cong \mathcal{T}\left(1-R^{3} / r^{3}\right) z \quad(r>R) .
$$

The pressure deviation $\delta p$ defined above Eq. (2.6) is negligibly small inside the droplet $\left(\propto R^{-2}\right)$ and is zero outside it for $g=0$ from Eqs. (3.4) and (3.12). The velocity field is expressed as

$$
\begin{aligned}
\boldsymbol{v}+v_{\mathrm{D}} \boldsymbol{e}_{z} & \cong v_{c}^{\prime} \frac{\hat{\eta}}{2}\left[\frac{R^{3}}{r^{3}} \boldsymbol{e}_{z}-\frac{3 R^{3}}{r^{5}} z \boldsymbol{r}\right] \quad(r>R) \\
& \cong v_{c}^{\prime}\left[\frac{z}{R^{2}} \boldsymbol{r}+\left(2+\frac{\hat{\eta}}{2}-\frac{2 r^{2}}{R^{2}}\right) \boldsymbol{e}_{z}\right] \quad(r<R),
\end{aligned}
$$

where $v_{c}^{\prime}$ is related to $v_{c}$ as

$$
v_{c}^{\prime}=\frac{1-\hat{\rho}}{1 / 3+\hat{\eta} / 2} v_{c}=-\frac{1}{T}\left(\frac{\partial T}{\partial p}\right)_{\mathrm{cx}} \frac{\lambda \mathcal{T}}{(1 / 3+\hat{\eta} / 2)} .
$$

Use has been made of the fact that the ratio $\left(1 / \rho^{\prime}\right.$ $-1 / \rho) /\left(s^{\prime}-s\right)$ is equal to the derivative along the coexistence curve $(\partial p / \partial T)_{\mathrm{cx}}$ (the Clausius-Clapeyron relation). Here we show the above velocity field for the gas droplet case with $\hat{\rho}=\hat{\eta}=0.3$ in Fig. 3 and for the liquid droplet case with $\hat{\rho}=\hat{\eta}=4$ in Fig. 4. We also show the temperature in Fig. 5 using Eq. (4.13). Similar temperature profiles have been found in simulation in the dynamic van der Waals theory [10].

To obtain these simple approximate solutions, we may omit $\gamma_{1} \delta T$ on the right-hand sides of Eqs. (3.10) and (3.11), because the interior temperature is almost homogenized. As a result, the interior thermal conductivity $\lambda^{\prime}$ does not appear in Eqs. (4.12)-(4.14). Furthermore, notice that $\boldsymbol{v}$ and $\delta T$ in this limit depend on the viscosities only through the ratio $\hat{\eta}$ $=\eta^{\prime} / \eta$. Hence, the same steady-state profiles follow even in the large limit of $\eta$ and $\eta^{\prime}$ with fixed ratio. This allows simulations with large viscosities or in the low Reynolds number limit [10].

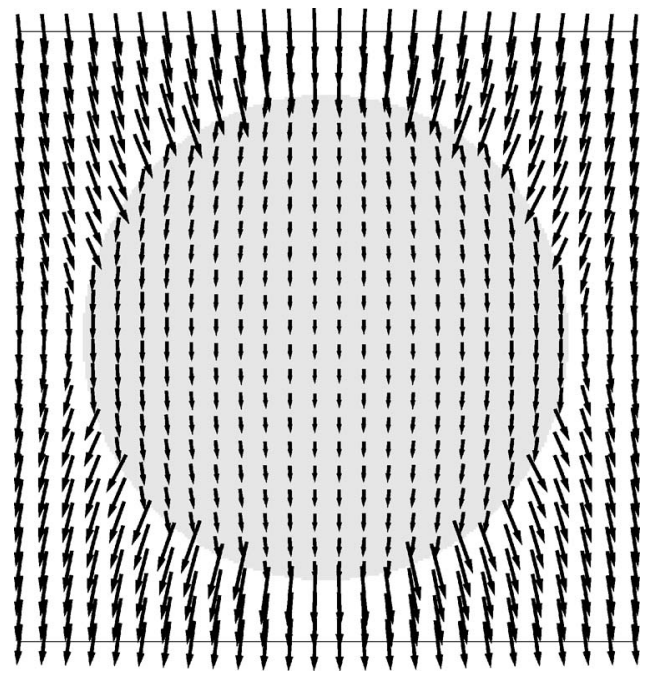

FIG. 4. Velocity field $\left(v_{x}, v_{z}\right)$ around a liquid droplet in a small temperature gradient with phase change in the reference frame moving with the droplet. Here $\rho^{\prime} / \rho=\eta^{\prime} / \eta=4$ and use is made of Eq. (4.14).

\section{Case of $g \neq 0$ and $\mathcal{T}=0$ with phase change}

Next we assume $g \neq 0$ and $\mathcal{T}=0$. The calculation may be performed as in the previous case. Not taking the large limit of $M_{1}$ and $M_{2}$ we may express $J_{1}$ as

$$
J_{1}=-\frac{(1+3 \hat{\eta} / 2) \hat{\rho}+5 \hat{\eta}}{(1+\hat{\eta})\left(M_{1} M_{2}+\epsilon_{2}\right)}(2+\hat{\lambda}) \rho^{\prime} v_{g}
$$

where $v_{g}$ is given by Eq. (1.1) and

$$
\epsilon_{2}=\frac{10 \hat{\eta} M_{1}}{2+3 \hat{\eta}}+20(1-\hat{\rho}) \frac{\hat{\eta}(2+\hat{\lambda})}{2+3 \hat{\eta}} .
$$

For large $M_{1}$ and $M_{2}$ we obtain $J_{1} / \rho^{\prime} \sim-v_{g} / M_{1} M_{2}$, and $v_{\mathrm{D}} \cong v_{g}$. The mass flux through the interface is very small. In this limit the interior temperature gradient is given by

$$
\left(\frac{d T}{d z}\right)_{\text {in }}=\mathcal{T}_{1} \cong-\frac{1}{3}\left(\frac{\partial T}{\partial p}\right)_{\mathrm{cx}}\left[\hat{\rho}+\frac{10 \hat{\eta}}{2+3 \hat{\eta}}\right] \rho g .
$$

The temperature inhomogeneity around the droplet is thus of order $(\partial T / \partial p)_{\mathrm{cx}} \rho g R$. Similarly, the interior pressure gradient is written as

$$
\left(\frac{d p}{d z}\right)_{\text {in }} \cong-g \rho^{\prime}-\frac{1}{3} g\left(\rho-\rho^{\prime}\right) \frac{10 \hat{\eta}}{2+3 \hat{\eta}} .
$$

\section{Near-critical case}

In the vicinity of the critical point the reduced temperature $\epsilon=1-T / T_{c}$ is small. We have the scaling behavior,

$$
\begin{aligned}
& M_{1} \cong A_{1} \epsilon^{1-\alpha-\beta} R / \xi, \\
& M_{2} \cong A_{2} \epsilon^{\alpha+\beta-1} R / \xi,
\end{aligned}
$$

where $A_{1}$ and $A_{2}$ are constants and $\xi=\xi_{-0} \epsilon^{-\nu}$ is the correlation length on the coexistence curve with $\xi_{-0}$ being a micro- 


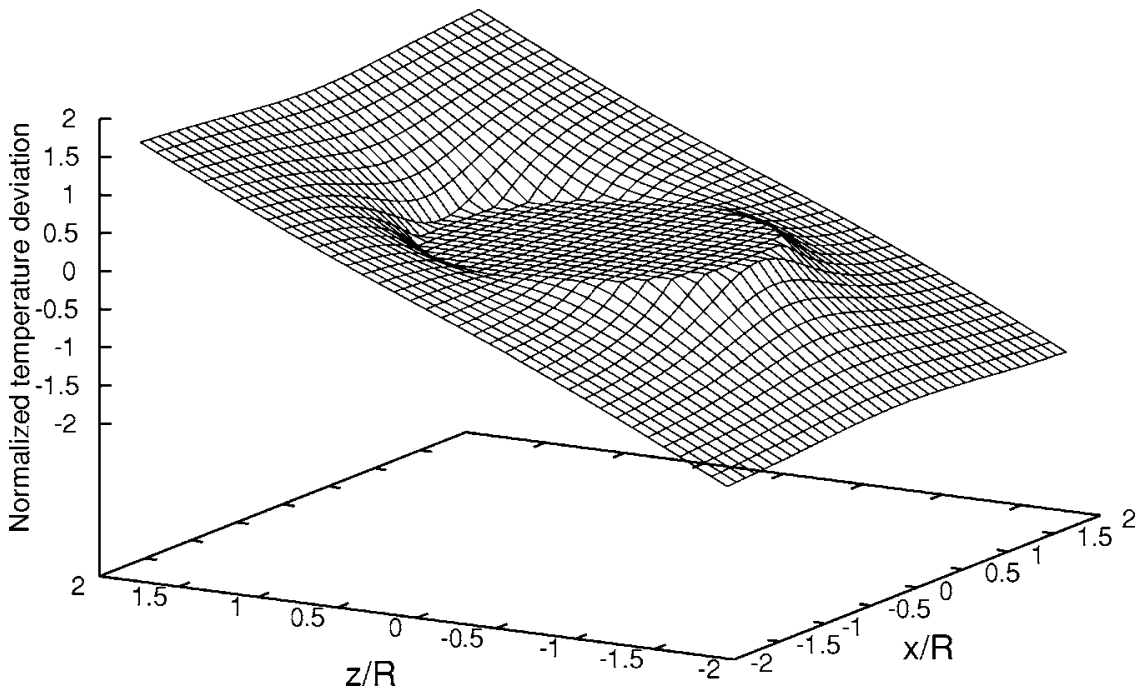

FIG. 5. Normalized temperature deviation $[T(\boldsymbol{r})-T(\mathbf{0})] / R \mathcal{T}$ with phase change in the $x-z$ plane. Its gradient vanishes within the droplet.

scopic length. The $\alpha \cong 0.11, \beta \cong 0.33$, and $\nu=(2-\alpha) / 3$ $\cong 0.63$ are the usual critical exponents, so $1-\alpha-\beta \cong 0.56$. Here $\Delta s \propto \epsilon^{\beta}, \gamma(=$ surface tension $) \sim k_{\mathrm{B}} T / \xi^{2} \propto \epsilon^{2 \nu}$, and $\gamma_{1} / \gamma$ $\cong 2 \nu /\left(T_{c}-T\right)$. We may assume $R \gg \xi$ since the interface thickness is of order $\xi$. We find

$$
M_{1} M_{2} \cong A_{0}(R / \xi)^{2}
$$

where the coefficient $A_{0}=A_{1} A_{2}=(\xi \rho \Delta s)^{2} T / \lambda \eta$ is a universal number estimated to be about 12 from the thermodynamic relations among the critical amplitudes and the relation $D_{T}=\lambda / \rho C_{p} \cong k_{\mathrm{B}} T / 6 \pi \eta \xi$ in the critical dynamics of fluids [28]. For $\mathrm{CO}_{2}$ we find $A_{2} \cong 0.21$ and $A_{1} \cong 12 / 0.21 \cong 57$.

From Eqs. (4.5)-(4.8) we may justify Eqs. (4.11)-(4.14) even if $M_{1}$ is not very large. From Eqs. (1.5) and (4.7) we estimate the velocities without gravity as

$$
\begin{gathered}
v_{\mathrm{D}} \cong\left(\frac{\partial T}{\partial p}\right)_{\mathrm{cx}} \frac{3 \lambda \mathcal{T}}{T\left(1-\rho^{\prime} / \rho\right)}=\frac{3 \lambda \mathcal{T}}{\rho T \Delta s} \\
v_{c}^{\prime} \cong-\frac{6}{5 T}\left(\frac{\partial T}{\partial p}\right)_{\mathrm{cx}} \lambda \mathcal{T} .
\end{gathered}
$$

The velocity field $\delta \boldsymbol{v}=\boldsymbol{v}+v_{\mathrm{D}} \boldsymbol{e}_{z}$ in the laboratory reference frame is smaller than the droplet velocity $v_{\mathrm{D}}$ by $\left(1-\rho^{\prime} / \rho\right) \sim \epsilon^{\beta}$ because it is of order $v_{c}^{\prime}$ from Eq. (4.14). In Fig. 6 we plot the scaled velocity $\delta \boldsymbol{v} / v_{c}^{\prime}=\left(v_{x} / v_{c}^{\prime}, \delta v_{z} / v_{c}^{\prime}\right)$ in the $x-z$ plane. More explicitly we obtain

$$
v_{\mathrm{D}} \cong A_{\mathrm{D}} \epsilon^{-x_{\mathrm{D}}} \mathcal{T} / T_{c}
$$

where $x_{\mathrm{D}}=4 / 3-\beta-2 \alpha / 3 \cong 0.96$. With $\mathcal{T} / T_{c}$ in $\mathrm{cm}^{-1}$ we find $A_{\mathrm{D}}=5 \times 10^{-5} \mathrm{~cm}^{2} / \mathrm{s}$ for $\mathrm{CO}_{2}$.

On earth, the gravity effect can be very strong. In fact, let $\mathcal{T}_{g}$ be the characteristic temperature gradient for which $v_{\mathrm{D}}$ in Eq. (4.23) is of order $v_{g}$. Some calculations yield the following estimate:

$$
\mathcal{T}_{g} \sim a_{g}(R / \xi)^{2}
$$

where $a_{g}=(\partial T / \partial p)_{s} \rho g \cong(\partial T / \partial p)_{\mathrm{cx}} \rho_{c} g$ is the adiabatic temperature gradient, equal to $0.27 \mathrm{mK} / \mathrm{cm}$ for $\mathrm{CO}_{2}$. If heated from above under $|\mathcal{T}| \gtrsim \mathcal{T}_{g}$, an upper fluid region will be in a one-phase state and the rest will be driven away from the critical point. If heated from below, the fluid will be in Rayleigh-Bénard turbulence at much smaller heat input [29]. Hydrodynamic turbulence in two-phase states is related to the problem of boiling and is itself a great challenge in future research $[26,30]$.

\section{SUMMARY AND REMARKS}

We have investigated thermocapillary hydrodynamics in the linear order around a spherical droplet, taking into account phase change at the interface, in one-component fluids. As a crucial ingredient of our theory, we have assumed the continuity of the temperature and the chemical potential across the interface as in Eqs. (2.15) and (2.16). The temperature gradient inside the droplet is then extremely small, as illustrated in Fig. 5. More general boundary conditions in Appendix B, which allow discontinuous changes in the temperature and the chemical potential, still lead to a

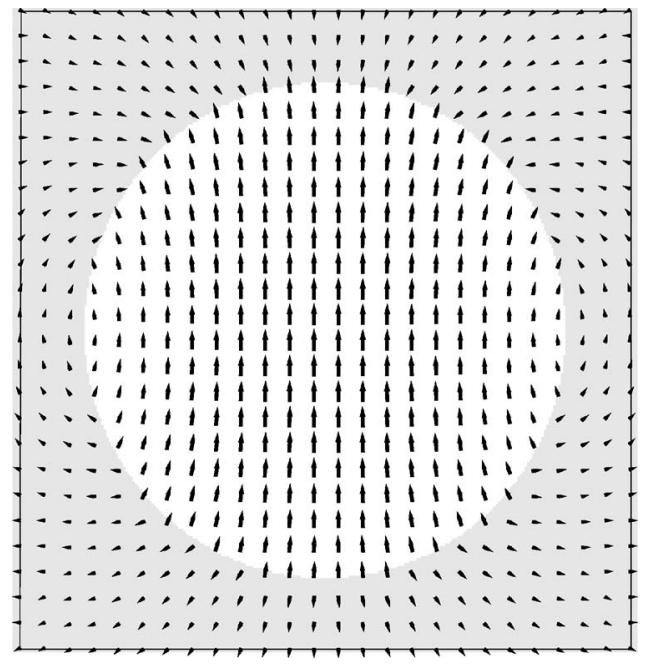

FIG. 6. Velocity field $\left(v_{x}, \delta v_{z}\right)$ in a small temperature gradient with phase change near the critical point in the reference frame fixed to the container. Here use is made of Eq. (4.14). 
very small interior temperature gradient as in Eq. (B6). This is because the latent heat transport dominates over the usual heat conduction within the droplet. We have found that our results can be much simplified if use is made of large sizes of the two dimensionless numbers $M_{1}$ Eq. (1.6) and $M_{2}$ in Eq. (4.4). The migration velocity is much smaller with phase change than without phase change by $M_{1}^{-1}$. It is towards the warmer region for a gas droplet and towards the cooler region for a liquid droplet, as can be seen in Eq. (4.7) or Eq. (4.12). A gas droplet can even touch a heated boundary wall that is wetted by liquid in equilibrium $[10,12]$, leading to apparent partial or complete wetting by gas in nonequilibrium.

Some further remarks are given below.

(i) Even on earth, we are interested in how a droplet reacts to heat flow and how the temperature changes inside it. In the presence of both heat flow and gravity, the internal temperature gradient is of order $(\partial T / \partial p)_{\mathrm{cx}} \rho g$ as in Eq. (4.18).

(ii) In space, a spherical bubble can be suspended in liquid in equilibrium at the center of a container. It is then of great interest how the velocity field and temperature evolve around the bubble after application of heat flux. Phase separation experiments in a small temperature gradient should also be interesting, where latent heat transport among the domains would enhance the Nusselt number [26]. For example, we may prepare a one phase state with a small temperature gradient and then cool both the top and bottom temperatures below $T_{c}$.

(iii) In this paper we have studied only steady solutions of the linearized hydrodynamic equations. We should examine time-evolution of two-phase states after application of heat flux, where adiabatic heat transport, called the piston effect, comes into play on rapid time scales [26,31]. We should note that there is a difference in the adiabatic temperature derivative $(\partial T / \partial p)_{s}$ between gas and liquid [26] and the temperature is changed differently within and without the droplet after a pressure change. As a result, a liquid (gas) droplet becomes cooler (warmer) than the surrounding fluid after application of heat flux. This temperature difference lasts for a long time in the case of a liquid droplet in gas because of the low thermal conductivity of gas [17].

(iv) Thermocapillary hydrodynamics is not still clearly understood near the critical point, where the singularities of the thermodynamic and dynamical properties largely influence the dynamics $[26,29,30]$.

(v) We are also interested in droplet motion in binary mixtures more generally and in helium systems such as $\mathrm{He}^{3}-\mathrm{He}^{4}$ mixtures at low temperatures [26]. Marek and Straub examined the effect of a small amount of noncondensable gas doped in the gas phase [11], which drastically alters thermocapillary convection. The Marangoni effect can come into play even at an extremely small density of the second component, on which we will report shortly.

\section{ACKNOWLEDGMENTS}

One of the authors (A.O.) thanks D. Beysens and V.S. Nikolayev for valuable discussions in an early stage of this work. Thanks are also due to D. Bedeaux for informative correspondence. This work was supported by Grants in Aid for Scientific Research and for the 21st Century COE project (Center for Diversity and Universality in Physics) from the Ministry of Education, Culture, Sports, Science and Technology of Japan.

\section{APPENDIX A: EQUILIBRIUM SPHERICAL DROPLET}

It is not trivial how a spherical gas (liquid) droplet can be suspended in metastable liquid (gas) as an equilibrium state at a fixed temperature $T_{0}$ below the critical temperature $T_{c}$ in the gravity-free condition [26]. We may exchange liquid and gas in the following discussion.

We fix the cell volume $V$ and the total mass at $V \rho_{0}$, where $\rho_{0}$ is the average mass density. With appearance of a droplet the mass density is $\rho^{\prime}$ inside it and $\rho$ outside it. The mass conservation gives

$$
v \rho^{\prime}+(V-v) \rho=V \rho_{0},
$$

where $v=4 \pi R^{3} / 3$ is the volume of the droplet with radius $R$ and the volume of the interface region is neglected. We then minimize the total Helmholtz free energy given by

$$
F=4 \pi \gamma_{0} R^{2}+v f\left(\rho^{\prime}\right)+(V-v) f(\rho),
$$

with respect to $\rho$ and $R$. Here $\gamma_{0}$ is the surface tension and $f(\rho)$ is the Helmholtz free energy density (at temperature $\left.T_{0}\right)$. From Eq. (A1) we obtain $\left(\partial \rho^{\prime} / \partial \rho\right)_{R}=1-V / v$ and $\left(\partial \rho^{\prime} / \partial v\right)_{\rho}=\left(\rho-\rho^{\prime}\right) / v$. The minimization condition $(\partial F / \partial \rho)_{R}=0$ readily gives

$$
\mu(\rho)=\mu\left(\rho^{\prime}\right),
$$

where $\mu(\rho)=\partial f(\rho) / \partial \rho$ is the chemical potential per unit mass. The other condition $(\partial F / \partial v)_{\rho}=0$ gives Laplace's law for the pressure difference,

$$
p\left(\rho^{\prime}\right)-p(\rho)=\frac{2 \gamma_{0}}{R}
$$

where $p=\rho \mu-f$ is the pressure.

Let $\rho_{\ell}$ and $\rho_{\mathrm{g}}$ be the liquid and gas densities, respectively, on the coexistence curve. For simplicity, we assume that $\rho_{0}$ is slightly smaller than $\rho_{\ell}$ (slight supercooling). Then $\rho \cong \rho_{0} \cong \rho_{\ell}, \rho^{\prime} \cong \rho_{\mathrm{g}}$, and $v \ll V$. In this case it is convenient to rewrite $\Delta F=F-V f_{0}$ as

$$
\begin{aligned}
\Delta F= & 4 \pi \gamma_{0} R^{2}+v\left[f\left(\rho^{\prime}\right)-f(\rho)-\mu_{0}\left(\rho^{\prime}-\rho\right)\right]+V\left[f(\rho)-f\left(\rho_{0}\right)\right. \\
& \left.-\mu_{0}\left(\rho-\rho_{0}\right)\right],
\end{aligned}
$$

where $\mu_{0}=\mu\left(\rho_{0}\right)$ and use has been made of Eq. (A1). To examine the second term we note the relations $f\left(\rho^{\prime}\right)=-p_{\text {cx }}$ $+\mu_{\mathrm{cx}} \rho^{\prime}+O\left(\left(\rho^{\prime}-\rho_{\mathrm{g}}\right)^{2}\right)$ and $f(\rho)=-p_{\mathrm{cx}}+\mu_{\mathrm{cx}} \rho+O\left(\left(\rho-\rho_{\ell}\right)^{2}\right)$, where $p_{\mathrm{cx}}$ and $\mu_{\mathrm{cx}}$ are the pressure and chemical potential, respectively, on the coexistence curve. The second term on the right-hand side of Eq. (A5) is then rewritten as $-v \delta \mu$ with

$$
\delta \mu \cong\left(\mu_{\mathrm{cx}}-\mu_{0}\right)\left(\rho-\rho^{\prime}\right) \cong\left(1-\rho_{\mathrm{g}} / \rho_{\ell}\right)^{2} \phi / K_{T},
$$

where $K_{T}=(\partial \rho / \partial \mu)_{T} / \rho^{2}=(\partial \rho / \partial p)_{T} / \rho$ is the isothermal compressibility in liquid and 


$$
\phi=\left(\rho_{\ell}-\rho_{0}\right) /\left(\rho_{\ell}-\rho_{\mathrm{g}}\right)
$$

is the initial supersaturation assumed to be a small positive number. The third term on the right hand side of Eq. (A5) is nearly equal to $V\left(\rho-\rho_{0}\right)^{2} / 2 \rho_{\ell}^{2} K_{T} \cong\left(1-\rho_{\mathrm{g}} / \rho_{\ell}\right)^{2} v^{2} / 2 V K_{T} \propto v^{2}$ $\propto R^{6}$. Thus,

$$
\Delta F \cong 8 \pi \gamma_{0} R_{\mathrm{c}}^{2}\left[\frac{x^{2}}{2}-\frac{x^{3}}{3}+\frac{1}{6}\left(\frac{R_{\mathrm{c}}}{R_{M}}\right)^{3} x^{6}\right],
$$

where $x=R / R_{\mathrm{c}}$ with $R_{c}=2 \gamma_{0} / \delta \mu$ being the usual critical radius for large $V$. The radius $R$ cannot exceed $R_{M}$ determined by $\phi V=(4 \pi / 3) R_{M}^{3}$. We notice that $\Delta F$ has a maximum at $R / R_{\mathrm{c}}=1+\left(R_{\mathrm{c}} / R_{M}\right)^{3}+\cdots$ and a minimum at

$$
R=R_{M}-\frac{1}{3} R_{\mathrm{c}}+\cdots
$$

for $R_{M} \sim(\phi V)^{1 / 3} \gg R_{c}$. The maximum corresponds to the unstable critical droplet and the minimum to the final equilibrium droplet we have sought.

\section{APPENDIX B: SURFACE DISSIPATION}

When the mass flux $J$ in Eq. (2.8) and the energy flux $J_{e}$ in Eq. (2.13) are nonvanishing through a gas-liquid interface, there can be small discontinuities in the temperature and the chemical potential, resulting in surface dissipation taking place in the interface region. From general thermodynamic arguments $[19,20]$ the corresponding entropy production rate $\sigma^{s}$ per unit area and per unit time is written as

$$
\sigma^{s}=J\left(\mu^{\prime} / T^{\prime}-\mu / T\right)+J_{e}\left(1 / T-1 / T^{\prime}\right) .
$$

Here the quantities in the interior (within a droplet) are primed, while those in the exterior (outside it) are unprimed. From the requirement that $\sigma^{s}$ should be nonnegative-definite, Onsager reciprocal relations may be postulated:

$$
\begin{gathered}
\mu^{\prime} / T^{\prime}-\mu / T=\alpha_{11} J+\alpha_{12} J_{e}, \\
1 / T-1 / T^{\prime}=\alpha_{12} J+\alpha_{22} J_{e} .
\end{gathered}
$$

The $J$ and $J_{e}$ are the fluxes from the interior to the exterior. The coefficients $\alpha_{11}, \alpha_{12}$, and $\alpha_{22}$ constitute a positivedefinite symmetric $2 \times 2$ matrix. The continuity of the temperature and the chemical potential assumed in Eqs. (2.15) and (2.16) can be obtained in the small limit of these coefficients. If the gas phase is dilute, the surface dissipation mainly occurs in the gas phase side within a distance of the mean-free-path $\ell_{\mathrm{mf}}$ inversely proportional to the mass density of the gas $\rho_{\mathrm{g}}$ (the Knudsen layer) [21-23]. From kinetic theory of gas we can make the following order estimations,

$$
\alpha_{11} \sim \frac{k_{\mathrm{B}}}{m \rho_{\mathrm{g}} v_{\mathrm{th}}}, \quad \alpha_{12} \sim \frac{m \alpha_{11}}{k_{\mathrm{B}} T}, \quad \alpha_{22} \sim \frac{m^{2} \alpha_{11}}{k_{\mathrm{B}}^{2} T^{2}},
$$

where $m$ is the molecular mass and $v_{\text {th }}=\left(k_{\mathrm{B}} T / m\right)^{1 / 2}$ is the thermal velocity.

In our droplet problem, let us calculate the effects of the surface dissipation in the limit of large $M_{1}$ and $M_{2}$ without gravity. The Knudsen number $\ell_{\mathrm{mf}} / R$ is assumed to be very small. Use of Eqs. (4.10) and (4.11) yields the surface discontinuities,

$$
\begin{gathered}
\delta \mu^{\prime}-\delta \mu \cong T\left[\alpha_{11}+\left(\mu+h^{\prime}\right) \alpha_{12}+\mu h^{\prime} \alpha_{22}\right] J_{1} \cos \theta, \\
\delta T^{\prime}-\delta T \cong T^{2}\left(\alpha_{12}+h^{\prime} \alpha_{22}\right) J_{1} \cos \theta
\end{gathered}
$$

where $J_{1} \cong 3 \rho^{\prime} v_{c}$. For the gas droplet case (where $\rho^{\prime}=\rho_{\mathrm{g}}$ ) the temperature discontinuity is of order

$$
\delta T^{\prime}-\delta T \sim \frac{q}{\rho^{\prime} v_{\text {th }} \Delta s} \sim \frac{\rho D_{T}}{\rho^{\prime} v_{\text {th }}} \mathcal{T},
$$

where $q=-\lambda \mathcal{T}$ is the applied heat flux and $D_{T}$ is the liquid thermal diffusivity. The interior temperature gradient is modified as

$$
\left(\frac{d T}{d z}\right)_{\text {in }}=\frac{3 \lambda \mathcal{T}}{\left(\rho^{\prime} \Delta s\right)^{2} T}\left[\frac{10 \eta^{\prime}(1-\hat{\rho})}{(1+3 \hat{\eta} / 2) R^{2}}+\frac{\alpha^{*}}{R}\right],
$$

where the first term in the brackets $\left(\propto R^{-2}\right)$ is the term obtained in Eq. (4.9) and the second term $\left(\propto R^{-1}\right)$ arises from the surface dissipation with

$$
\alpha^{*}=\left[\alpha_{11}+\left(h+h^{\prime}\right) \alpha_{12}+h h^{\prime} \alpha_{22}\right]\left(\rho^{\prime}\right)^{2} T .
$$

For the gas droplet case we have $\eta^{\prime} \sim m v_{\text {th }} / a^{2}$ and $\alpha^{*} \sim v_{\text {th }} \rho_{\mathrm{g}}$, where $a$ is the molecular radius. In Eq. (B5) the new second term $\left(\propto R^{-1}\right)$ dominates over the first term ( $\left.\propto R^{-2}\right)$ for $R \gg \ell_{\mathrm{mf}}=m / a^{2} \rho_{\mathrm{g}}$.

\section{APPENDIX C: AXISYMMETRIC VELOCITY FIELD}

When a spherical droplet is considered, we may express the velocity field $\boldsymbol{v}$ generally in terms of three scalar functions $H, Q$, and $S$ as [26]

$$
\boldsymbol{v}(\boldsymbol{r})=\nabla H+Q \boldsymbol{r}+(\boldsymbol{r} \times \nabla) S
$$

without loss of generality. We use the spherical coordinates $(r, \theta, \varphi)$. The condition $\nabla \cdot \boldsymbol{v}=0$ in Eq. (2.3) then gives

$$
\nabla^{2} H+3 Q+r Q^{\prime}=0
$$

where $\quad Q^{\prime}=\partial Q / \partial r$. The velocity components $v_{i}=\boldsymbol{e}_{i} \cdot \boldsymbol{v}(i=1,2,3)$ along the three-unit vectors in Eq. (2.1) are expressed as

$$
\begin{gathered}
v_{1}=H^{\prime}+Q r, \quad v_{2}=\frac{1}{r} \nabla_{\theta} H-\nabla_{\varphi} S, \\
v_{3}=\frac{1}{r} \nabla_{\varphi} H+\nabla_{\theta} S,
\end{gathered}
$$

where $H^{\prime}=\partial H / \partial r$ and

$$
\begin{gathered}
\nabla_{\theta} \equiv \partial / \partial \theta=r \boldsymbol{e}_{2} \cdot \nabla, \\
\nabla_{\varphi} \equiv(\sin \theta)^{-1} \partial / \partial \varphi=r \boldsymbol{e}_{3} \cdot \nabla .
\end{gathered}
$$

We may express $H, Q$, and $S$ as linear combinations of the spherical harmonic functions $Y_{\ell m}(\theta, \varphi)$. As a merit of this representation, if the equations for $\boldsymbol{v}$ are written in terms of $H, Q$, and $S$, the terms with different $\ell$ and $m$ are not mixed in the linear order. However, mixing occurs if $v_{1}, v_{2}$, and $v_{3}$ are expressed as linear combinations of $Y_{\ell m}(\theta, \varphi)$. 
In the axisymmetric case $\boldsymbol{v}$ is independent of $\varphi$, leading to $v_{3}=0$. We may set

$$
H=\hat{H}(r) \cos \theta, \quad Q=\hat{Q}(r) \cos \theta, \quad S=0 .
$$

Eq. (C2) is rewritten as $\mathcal{L}_{1} \hat{H}+3 \hat{Q}+r \hat{Q}^{\prime}=0$ with

$$
\mathcal{L}_{1}=\partial^{2} / \partial r^{2}+(2 / r) \partial / \partial r-2 / r^{2},
$$

where $\nabla^{2} h=\cos \theta \mathcal{L}_{1} \hat{H}$ and $\hat{Q}^{\prime}=d \hat{Q} / d r$. From the symmetry the pressure deviation may also be set

$$
\delta p=\delta \hat{p}(r) \cos \theta .
$$

From $\nabla^{2} \delta p=0$ we find $\mathcal{L}_{1} \delta \hat{p}=0$ in the bulk region $(r \neq R)$. leading to the power law behavior,

$$
\delta \hat{p}=p_{1} r+p_{2} \frac{1}{r^{2}}(r>R)=p_{1}^{\prime} r(r<R) .
$$

Using Eq. (C2) and $S=0$ we find

$$
\boldsymbol{e}_{1} \cdot \nabla^{2} \boldsymbol{v}=-\frac{2 Q}{r}, \quad \boldsymbol{e}_{2} \cdot \nabla^{2} \boldsymbol{v}=-\frac{\partial}{\partial \theta}\left(\frac{Q}{r}+Q^{\prime}\right) .
$$

Then the momentum equation, Eq. (2.4), gives

$$
\frac{2 \eta}{r} \hat{Q}+\frac{\partial}{\partial r} \delta \hat{p}+\rho g=0
$$

$$
\eta\left(\frac{1}{r} \hat{Q}+\hat{Q}^{\prime}\right)+\frac{1}{r} \delta \hat{p}+\rho g=0
$$

for $r>R$. The same equations hold for $r<R$ if $\eta$ and $\rho$ are replaced by $\eta^{\prime}$ and $\rho^{\prime}$, respectively. Elimination of $\delta \hat{p}$ gives $\mathcal{L}_{1} \hat{Q}=0$, leading to Eqs. (3.2) and (3.3). The pressure deviation is written as in Eq. (3.4).

To impose the boundary conditions at $r=R$, we need to calculate the following components of the shear strain tensor in Eq. (2.12):

$$
\boldsymbol{e}_{1} \cdot \stackrel{\leftrightarrow}{e} \cdot \boldsymbol{e}_{1}=\left(-\frac{4}{r} \hat{H}^{\prime}+\frac{4}{r^{2}} \hat{H}-4 \hat{Q}\right) \cos \theta
$$

$$
\boldsymbol{e}_{2} \cdot \overleftrightarrow{e} \cdot \boldsymbol{e}_{1}=-\left(\frac{2 \hat{H}^{\prime}}{r}-\frac{2 \hat{H}}{r^{2}}+\hat{Q}\right) \sin \theta
$$

Eqs.(3.10) and (3.11) can then be obtained.
[1] B. Levich, Physicochemical Hydrodynamics (Prentice-Hall, Englewood Cliffs, N. J., 1962).

[2] J. Hadamard, C. R. Acad. Sci. III Paris 152, 1735 (1911).

[3] D. Rybczynski, Bull. Int. Acad. Sci. Cracovie 1, 40 (1911).

[4] N. O. Young, J. S. Goldstein, and M. J. Block, J. Fluid Mech. 6, 350 (1959). If heated from below, the balance $v_{g}=-v_{\mathrm{Y}}$ yields $R \sim \gamma_{1}|\mathcal{T}| / \rho g$. In this experiment $\gamma_{1} \sim 0.1 \mathrm{dyne} / \mathrm{cmK}$ and $\rho \sim 1 \mathrm{~g} / \mathrm{cm}^{3}$, so $R \sim 10^{-4}|\mathcal{T}| \mathrm{cm}$ with $\mathcal{T}$ in $\mathrm{K} / \mathrm{cm}$ was obtained on earth. The Marangoni number [5] was much smaller than 1 for $R$ realized.

[5] The Marangoni number Ma is the typical ratio of the nonlinear convection term $\boldsymbol{v} \cdot \nabla \delta T$ to the diffusion term $D_{T} \nabla^{2} \delta T$ in the heat conduction equation in surface-tension-driven flow.

[6] G. Wozniak, R. Balasubramaniam, P. H. Hadland, and R. S. Subramanian, Exp. Fluids 31, 84 (2001); In this space experiment $\mathcal{T} \sim 0.025 \mathrm{~K} / \mathrm{cm}, R \sim 0.6 \mathrm{~cm}$, and the bubble velocity was $0.036 \mathrm{~cm} / \mathrm{s}$, but $\mathrm{Ma}\left(\propto R^{2} \mathcal{T}\right)$ was very large $(\sim 452)$ due to large $R$.

[7] N. Ichikawa, M. Kawaji, and M. Misawa, Microgravity Sci. Technol. 7, 156 (2003).

[8] B. Braun, Ch. Ikier, H. Klein, and D. Woermann, Chem. Phys. Lett. 233, 565 (1995).

[9] D. Beysens, Y. Garrabos, V. S. Nikolayev, C. Lecoutre-Chabot, J.-P. Delville, and J. Hegseth, Europhys. Lett. 59, 245 (2002).

[10] A. Onuki, Phys. Rev. Lett. 94, 054501 (2005).

[11] R. Marek and J. Straub, Int. J. Heat Mass Transfer 44, 619 (2002).

[12] Y. Garrabos, C. Lecoutre-Chabot, J. Hegseth, V. S. Nikolayev, D. Beysens, and J.-P. Delville, Phys. Rev. E 64, 051602 (2001).

[13] A. Michels, J. V. Sengers, and P. S. van der Gulik, Physica (Amsterdam) 28, 1216 (1962). This report shows $\lambda / k_{\mathrm{B}}=6.0$ $\times 10^{19} \mathrm{~cm} / \mathrm{s}$ in liquid on the coexistence curve at $T=25 \mathrm{C}$, where the gas and liquid densities are 0.25 and $0.73 \mathrm{~g} / \mathrm{cm}^{3}$. Here, we have $\Delta s / k_{\mathrm{B}}=3.4 \times 10^{22} \mathrm{~g}$ from the ClausiusClapeyron relation with $(\partial p / \partial T)_{\mathrm{cx}} / k_{\mathrm{B}}=1.2 \times 10^{22} \mathrm{~cm}^{3}$.

[14] J. Luettmer-Strathmann, J. V. Sengers, and G. A. Olchowy, J. Chem. Phys. 103, 7482 (1995); For $\mathrm{CO}_{2}$ this work indicates that the first relation in Eq. (1.7) holds away from the critical point, while $a$ there is replaced by the correlation length $\xi$ as the critical point is approached.

[15] You-Xiang Zuo and E. H. Stenby, Fluid Phase Equilib. 132, 139 (1997); S. B. Kiselev and J. F. Ely, J. Chem. Phys. 119, 8645 (2003); The mean-field expression of the surface tension $\gamma \sim k_{\mathrm{B}} T\left(1-T / T_{c}\right)^{3 / 2} / a^{2}$, is a rather good approximation over a wide temperature region (not very close to the critical point).

[16] In the axisymmetric case the interface position is written as $r$ $=R+\zeta \cos \theta$, in the linear order. The droplet is displaced by $\zeta$, along the $z$ axis, but remains spherical.

[17] R. B. Bird, W. E. Stewart, and E. N. Lightfoot, Transport Phenomena (Wiley, New York, 2002).

[18] L. D. Landau and E. M. Lifshitz, Fluid Mechanics (Pergamon, New York, 1959).

[19] D. Bedeaux, A. M. Albano, and P. Mazur, Physica A 82, 438 (1976); D. Bedeaux, Adv. Chem. Phys. 64, 47 (1986).

[20] E. Johannessen and D. Bedeaux, Physica A 336, 252 (2004).

[21] Y. P. Pao, Phys. Fluids 14, 306 (1971).

[22] Y. Sone and Y. Onishi, J. Phys. Soc. Jpn. 35, 1773 (1973).

[23] G. Fang and C. A. Ward, Phys. Rev. E 59, 417 (1999); Phys. Rev. E 59, 441 (1999).

[24] F. Graner, R. M. Bowley, and P. Nozières, J. Low Temp. Phys. 80, 113 (1990).

[25] W. van Saarloos and J. D. Weeks, Phys. Rev. Lett. 74, 290 (1995). 
[26] A. Onuki, Phase Transition Dynamics (Cambridge University Press, Cambridge, 2002); see Appendix 10E for general expressions of vectors in terms of the spherical harmonic functions. See Eq. (2.2.37) for $(\partial T / \partial p)_{s}$ in gas and liquid.

[27] The velocity field of a moving sphere is given by $\boldsymbol{v}$ $=\left(3 v_{\mathrm{D}} R / 4 r\right)\left[\boldsymbol{e}_{z}+r^{-2} z \boldsymbol{r}\right]+\left(v_{\mathrm{D}} R^{3} / 4 r^{3}\right)\left[\boldsymbol{e}_{z}-3 r^{-2} z \boldsymbol{r}\right]-v_{\mathrm{D}} \boldsymbol{e}_{z}$ in the moving reference frame under the stick boundary condition [18]. In this case we have $Q_{1}=3 u_{0} / 2$ and $H_{1}=u_{0} / 4$, in Eq. (3.2).

[28] The critical phenomena of fluids can be mapped onto those of the Ising spin systems [26]. In the latter systems let $m\left(\propto \epsilon^{\beta}\right)$ and $\chi^{\prime}\left(\propto \epsilon^{-\gamma}\right)$ be the average spin and the susceptibility on the coexistence curve. Then, if we set $\lambda \eta=k_{\mathrm{B}} T \rho C_{p} / 6 \pi \xi$, we obtain $A_{0}=24 \pi \xi^{3} m^{2} / \chi^{\prime} \cong 12$, using the relations among the critical amplitudes.

[29] A. B. Kogan, D. Murphy, and H. Meyer, Phys. Rev. Lett. 82, 4635 (1999); A. Furukawa and A. Onuki, Phys. Rev. E 66, 016302 (2002).

[30] A. Onuki, Physica A 314, 419 (2002).

[31] A. Onuki, H. Hao, and R. A. Ferrell, Phys. Rev. A 41, R2256 (1990); A. Onuki and R. A. Ferrell, Physica A 164, 245 (1990). 